\section{Reticular Chemistry: Occurrence and Taxonomy of Nets and Grammar for the Design of Frameworks}

\author{
NATHAN W. OCKWIG, ${ }^{\dagger}$ \\ OLAF DELGADO-FRIEDRICHS, \\ MICHAEL O'KEEFFE, ${ }^{*, \neq}$ AND OMAR M. YAGHI ${ }^{*, \dagger}$ \\ Materials Design and Discovery Group, \\ Department of Chemistry, University of Michigan, \\ Ann Arbor, Michigan 48109, and Department of Chemistry \\ and Biochemistry, Arizona State University, \\ Tempe, Arizona 85287
}

Received October 6, 2004

\section{ABSTRACT}

The structures of all 1127 three-periodic extended metal-organic frameworks (MOFs) reported in the Cambridge Structure Database have been analyzed, and their underlying topology has been determined. It is remarkable that among the almost infinite number of net topologies that are available for MOFs to adopt, only a handful of nets are actually observed. The discovery of this inversion between expected and observed nets led us to deduce a system of classification "taxonomy" for interpreting and rationalizing known MOF structures, as well as those that will be made in future. The origin of this inversion is attributed to the different modes with which MOF synthesis has been approached. Specifically, three levels of complexity are defined that embody rules "grammar" for the design of MOFs and other extended structures. This system accounts for the present proliferation of MOF structures of high symmetry nets, but more importantly, it provides the basis for designing a building block that "codes" for a specific structure and, indeed, only that structure.

"Fossils have long been studied as great curiosities, collected with great pains, treasured with great care and at a great expense, and showed and admired with as much pleasure ... and this has been done by thousands who have never paid the least regard to that wonderful order and regularity with which Nature has disposed of these singular productions and assigned to each class its particular stratum". These are the words of William Smith,

Nathan W. Ockwig was born in Granite Falls, MN (1973). He received his B.S. in chemistry from St. Cloud State University (1996) and M.S. from the University of Michigan (2001). At present, he is a doctoral candidate in the Department of Chemistry of the University of Michigan with Professor Omar M. Yaghi and his primary focus is the design and characterization of porous crystalline materials.

Olaf Delgado-Friedrichs was born in Ibbenbüren, Germany (1963). He received his University Diploma (1990), Ph.D. (1994) and Habilitation (2002) from Bieefeld University. He has served as a Faculty Research Associate with Professor O'Keeffe at Arizona State University, 2000-2002, and again since October 2004. In 2002-2004, he joined Professor Daniel Huson's research group on Algorithms in Bioinformatics at Tubingen University. He is a mathematician by training and focuses on applications of discrete mathematics, in particular discrete geometry, to such fields as solid-state chemistry and molecular biology.

Michael O'Keeffe was born in Bury St Edmunds, England (1934). He received his B.Sc. (1954), Ph.D. (1958), and D.Sc. (1976) from the University of Bristol. He is Regents' Professor of Chemistry at Arizona State University, where he has been since 1963. His current research is particularly focused on studying beautiful patterns found in chemistry and elsewhere. now known as the father of modern geology, written on January 5, 1796, as he began to describe how fossils can be traced to different layers of rocks. This remarkable discovery led him to paint the world's first geologic map: that of England and Wales. ${ }^{1}$

We draw special inspiration from William Smith's statement as his work endeavored to find organizing principles for seemingly disparate observations. Witnessing the recent plethora of reports on extended structures especially those of metal-organic compositions, one cannot help but feel similarly struck by their beauty and diversity. Nearly 12000 extended metal-organic compounds have been documented in the Cambridge Structure Database (CSD). ${ }^{2}$ Although we and others have summarized some important preparative and functional features of these compounds,,$^{3-9}$ very little has been done to develop a rationalization of their structure and to answer the bigger question of how these structures and other extended structures can be truly constructed by design. ${ }^{10}$ In this Account, we present organizing principlestaxonomy-for extended structures and illustrate their use in defining the rules-grammar-for their logical construction.

The development of such means to construct chemical structures has always had central importance in chemistry. They have been successfully achieved in the synthesis of organic molecules by a process referred to as retrosynthesis with profound impact on pharmaceutical research and technology. ${ }^{11}$ On the other hand, an analogous system of principles and general preparative processes (protocols) does not exist for achieving the logical synthesis of extended structures or for prediction of their underlying connectivity (topology). There are two reasons for this state of affairs: First, unlike the step-by-step synthesis of organic molecules, which allows sequential building up of a particular structure, the synthesis of extended structures must be done as a one step reaction due to insolubility of products. However, it is possible to observe kinetically favored intermediary MOFs in route to the thermodynamically favored product. ${ }^{12}$ This precludes the use of approaches of the retrosynthesis type for the construction of extended structures and necessitates that their synthesis be done by a trial-and-error procedure

\footnotetext{
* Corresponding authors. E-mail addresses: mokeeffe@asu.edu oyaghi@umich.edu.

$\dagger$ University of Michigan.

‡ Arizona State University.
}

Omar M. Yaghi was born in Amman, Jordan (1965). He received his B.S. in chemistry from the State University of New York-Albany (1985) and his Ph.D. from the University of Illinois-Urbana (1990) with Professor Walter G. Klemperer. From 1990 to 1992, he was an NSF Postdoctoral Fellow at Harvard University with Professor Richard H. Holm. He joined the faculty at Arizona State University in 1992. He was awarded the ACS-Exxon Solid-State Chemistry Award in 1998. In June 1999, he moved to the University of Michigan as a Professor of Chemistry. More recently, he was awarded the Robert W. Parry Collegiate Chair by the Chemistry Department at UM and the Sacconi Medal by the Inorganic Division of the Italian Chemical Society. He has established several research programs dealing with the reticular synthesis of discrete polyhedra and extended frameworks from organic-inorganic building blocks. 
rather than by a deliberate step-by-step process. Such a procedure, although useful, does not lend itself well to directed synthesis. Second, without knowledge of the possible structures that could result from the assembly of starting molecular building blocks and without conceptual strategies that could be used to rationalize such structures and predict new ones, it is difficult or impossible to develop a basis for their designed construction.

Although progress in addressing these two issues will have a tangible impact on many areas of chemistry concerned with extended structures, in this Account we focus primarily on a rapidly growing subset of structures, metal-organic frameworks (MOFs). In general, MOF structures have two main components: the organic linkers and the metal ions. The linkers act as "struts" that bridge metal centers, which in turn act as "joints" in the resulting MOF architecture. This class of materials has received widespread attention due to the diversity of linkers and metal ions that can be employed and the fact that the products of their assembly can be crystallized and fully characterized. In recent contributions, we reported how the concept of secondary building units (SBUs) is being applied with eminent success to the design of highly porous and rigid MOF structures. ${ }^{13,14}$ Here, clusters such as those of the copper-carboxylate paddlewheel, $\mathrm{Cu}_{2}\left(\mathrm{O}_{2} \mathrm{C}-\right)_{4}$, and the octahedral basic zinc-carboxylate, $\mathrm{Zn}_{4} \mathrm{O}\left(\mathrm{O}_{2} \mathrm{C}-\right)_{6}$, have been used as rigid SBUs that, respectively, act as square and octahedral joints (i.e., vertices) in the framework. If the designer identifies the one step reaction conditions that reproducibly lead to such a particular SBU, then control of the vertex geometry in the resulting MOF is possible. Since the organic links remain intact and their geometry is preserved throughout the assembly process, one should also be able to predict the underlying topology of the resulting MOF structure.

However, the number of topologies that could, in principle, result from linking molecular shapes into extended MOF structures is vast, giving rise to three questions: (1) From this large topology space, how do we as designers identify the most important topologies that should be considered in this chemistry? (2) How are they distributed among crystal structures already reported in the CSD? (3) How can these data be interpreted, organized, and classified for the purpose of developing systems of grammar and taxonomy that can lead to the design and construction of extended structures, the rationalization of existing structures, and the prediction of new ones?

To address these questions, we have undertaken a study involving the analysis of all 1127 crystal structures reported in the CSD up to October 2003 in which metal ions are coordinated to organic links to form 3-D connected structures.

\section{MOF Crystal Structures in CSD and Their Nets}

Within the last 30 years, reports of MOF crystal structures have increased at an exponential rate with nearly 1500 structures reported just last year alone (Figure 1A). Indeed, since 1978 the number of MOF structures reported has

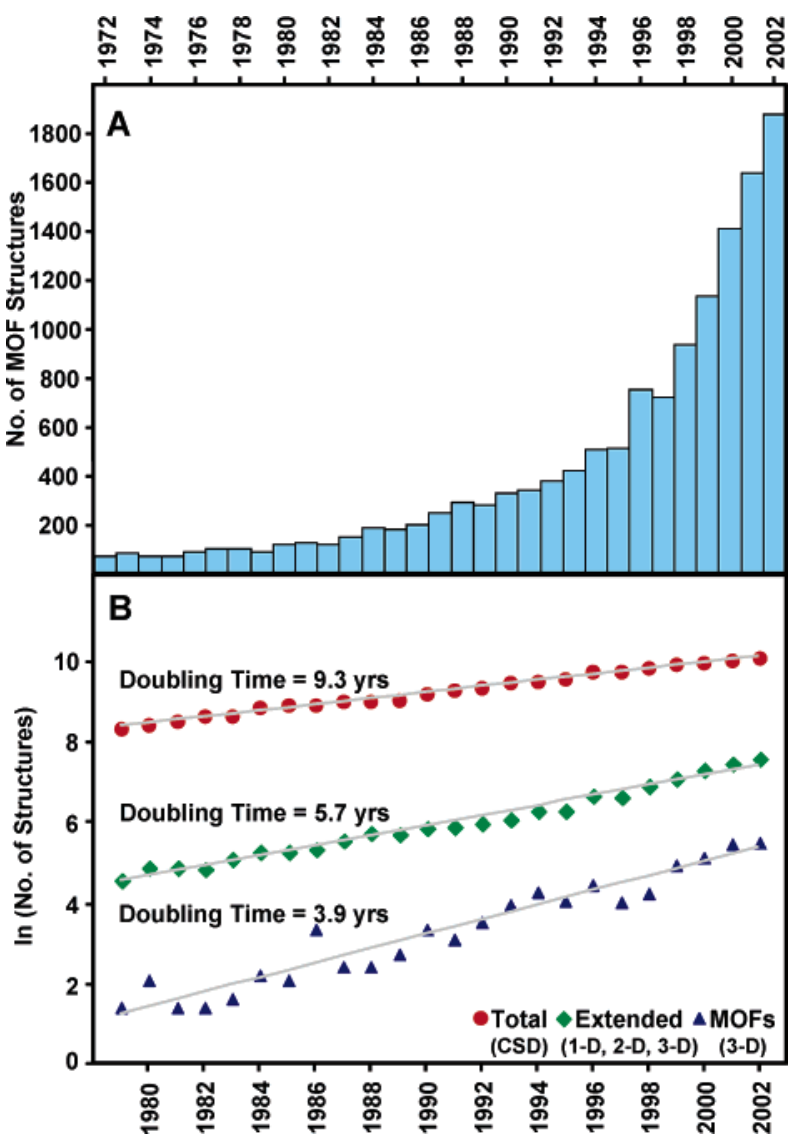

FIGURE 1. Progression (a) of metal-organic framework (MOF) structures reported in the CSD from 1972 through 2002 illustrating the dramatic increase in reports; and the natural log of number of structures (b) plotted as a function of time, showing the doublingtimes of total structures (entire CSD), extended MOF structures (1D, 2-D, and 3-D), and 3-D MOFs.

doubled every 3.9 years for 3-D periodic structures and every 5.7 years for all MOFs (1-D, 2-D, and 3-D structures). This is compared to a doubling time of 9.3 years for the entire CSD (Figure 1B). This disproportionate growth reflects the emerging importance of MOFs in science and technology. It is interesting to note that one can say the same thing about MOF crystals today that William Smith said of fossils over 200 years ago: extensive efforts applied to their discovery have not been accompanied by an equal effort striving to systematize their structures and to develop a cohesive set of principles for rationalizing their construction.

We believe that knowledge of the topology of MOF structures plays an essential role in their simplification and subsequent systematization. Accordingly, we have determined, using established methods, ${ }^{14,15}$ the topology of each MOF having a 3-D periodic structure reported in the CSD. First, the chemical moieties that represent the vertices (branching points) and those that connect them (links) were identified. The crystal structure was reduced to an irreducible net topology in which points representing the vertices are linked by lines representing the links. ${ }^{16}$ We were aided by the computer program "Systre", which uniquely and unambiguously determines the topology of 
Building Blocks
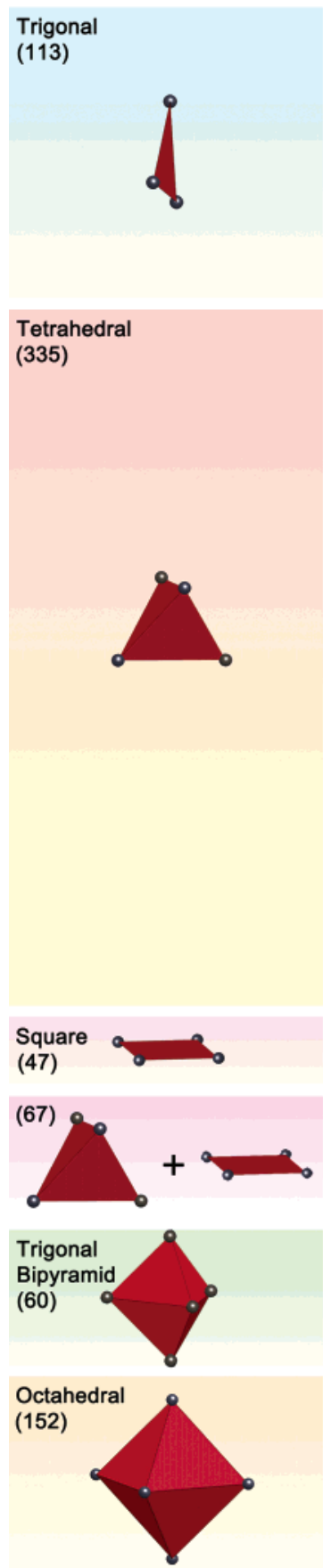

CSD Refcode

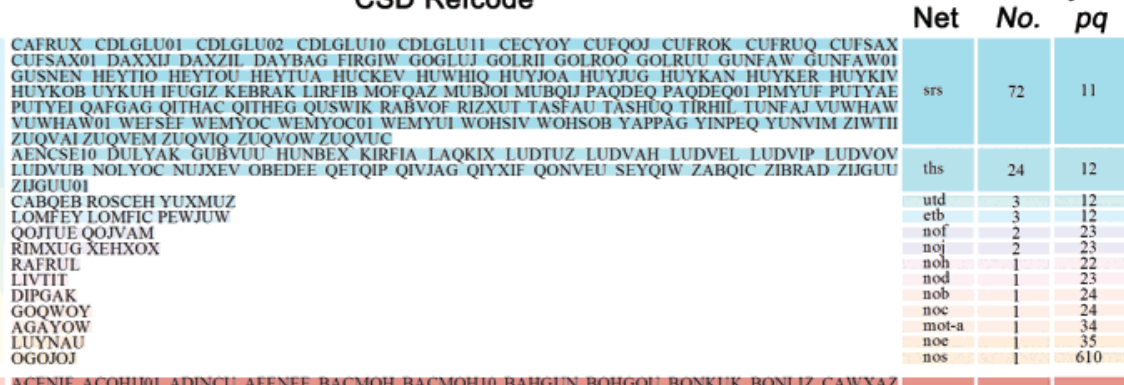

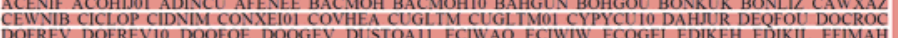

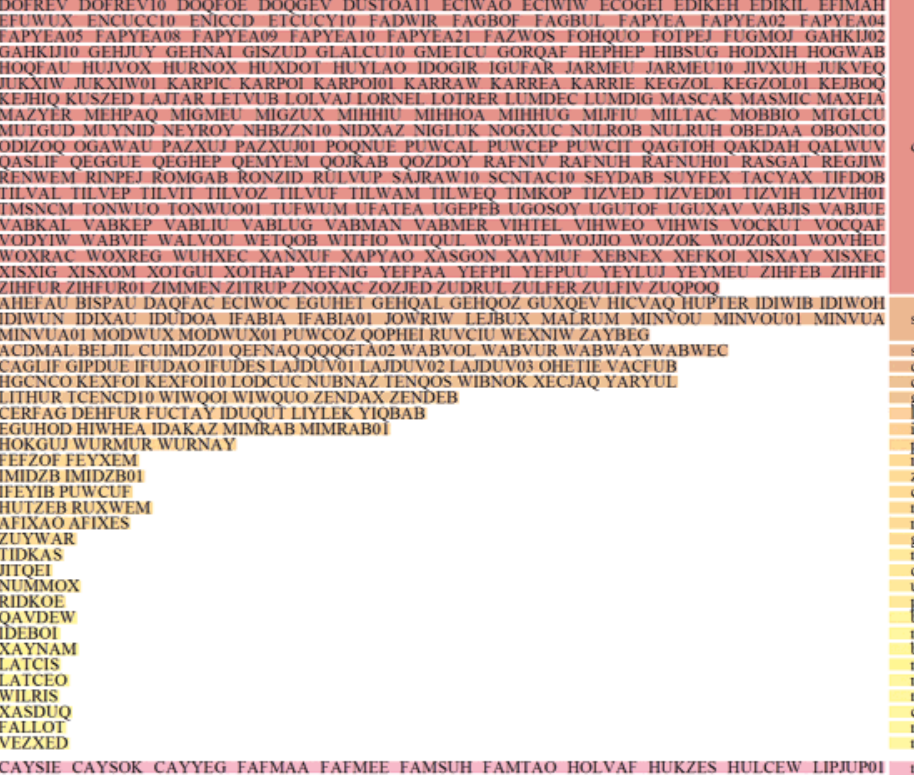

CAYSIE CAYSOK CAYYEG FAFMAA FAFMEE FAMSUH FAMTAO HOLVAF HUKZFS HULCEW LIPJUPOI

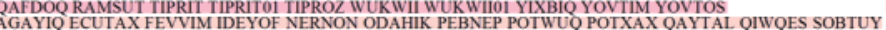
TISAG YOPRAR TUSGOD UGENUP VUGBOO ZURROT
AZPYCD ISGOH MEGFIN MIMVEJUFU QIV VIW XUU XENCAU ZNACEU

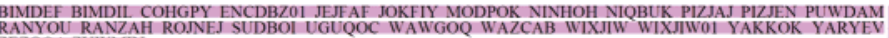

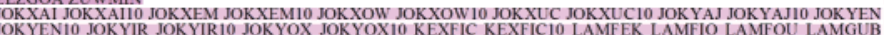
OKKYN10 JOKYIR JOKYIRI0 JOKYOX JOKYOXI0 KEXFIC KEXYCTO LAMFE LAMFHO LAMFOU LAMGUB MGI

BUKLOJ BUKLOJO1 CIIXIE DOSYAL GUGWOUOI GUXQAR JOSNAG JOSNAGO1 KEXTAI KEXIEM LESBIU WUBMA I APWIN QAYIOZ OIJLAW OOZJOE QUHYEXO1 QUPZAC QUPZIK QUPZOQ SIVJAI TIJNIJ WOPXXE FESOK BALA VVO1 DAOSOOD MABNE I NGHOA NIVFUT NIVFUTO1 QUJVEW TUTLEZ WOPXAA WOPXAA01 XAGIFE XANZUH XAPBAR XAPBEV XIPHIN XUHSOIYEQGIK
MABJOP MABUV MABJUVOI MABKEG MABKIK MABKIKO1 AAUBOPACUBOPOI XIZPIF
WUGSUM WUGSUMOI QIBTAW
COYYOE

ABUWOJ ACEPON AFEHUO AFEKAX BUJKEWO1 BUJWAF BUJWAFO1 BUJWAFO2 BUKBUE CAHMON CAHMUT

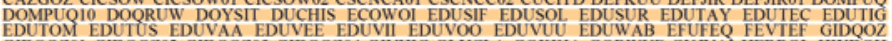
GIDOOZO1 GIDOOZO2 GIDOOZZ3 GIDOOZO4 GIVHIC GOYCLA GOKKIA GORWUIF GUJJVAI HERROL GIIXDOH

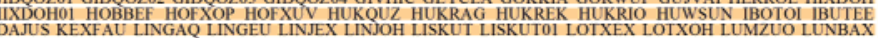
NBEB LON MABLD MACUFR MEBFAA MESAL MIHBAG MJAA MITXW MIZOOB MOLI NOBLOF NOBLUL NOBMAS NOLOUA ODUPUY OFUWIVO1 OFUWIVO2 OXCFOR PURSOK PUWDEO QANXI

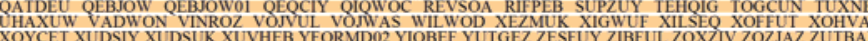
XOYCEI XUDSIY XUDSUK XUVHEB YFORMDO2 YIQBEF YUTGEZ ZESFUY ZIBFUL ZOXZIV ZOZJAZ ZUTBAR
JTINIIJ JEINOL QUSBIP VARREL VARRIP ZUVIEP
EGOZB

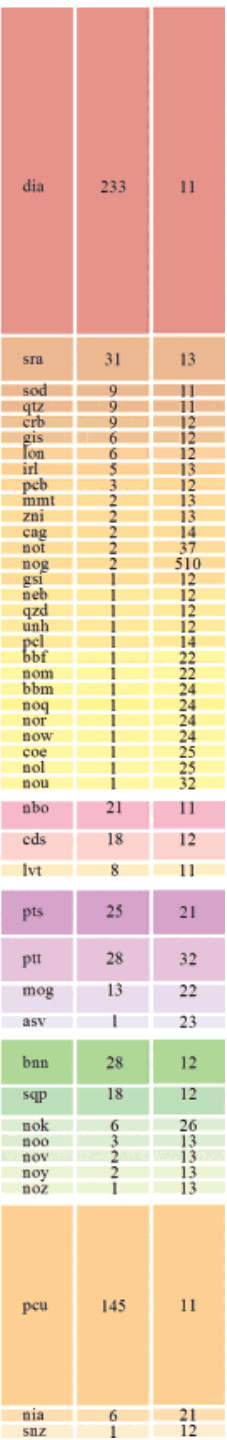

FIGURE 2. Distribution of net types in the CSD shown (from left) for each building block geometry and refcode of structures (center) in CSD and their corresponding net taxonomy (on right) as classified by net type, frequency of net occurrence in MOFs, and transitivity (pq).

any 3-D net. ${ }^{17}$ The results of this analysis are presented in the following section and illustrated in Figure 2.

\section{Recurrence of Nets with High Symmetry and Their Taxonomy}

Among the 1127 MOF structures analyzed in this study, 774 are reduced to topologies belonging to nets with vertices having one coordination number, $\mathrm{CN}$. These nets will be the primary focus of this article including those having tetrahedral and square vertices in the same net. The remaining MOFs (353) having mixed coordination numbers $(3,4 ; 3,5 ; 4,5 ; 3,6 ; 4,6 ; 5,6)$ will be considered in a future publication.

The large majority of 3-D MOFs with uninodal net topology have tetrahedral geometry $(\mathrm{CN}=4 ; 335,43.3 \%)$, followed by octahedral $(\mathrm{CN}=6 ; 152,19.6 \%)$ and triangular $(\mathrm{CN}=3 ; 113,14.6 \%)$ geometries (Figure 2). Significantly fewer MOF structures have square $(\mathrm{CN}=4 ; 47,6.1 \%)$, 
mixed square and tetrahedral $(\mathrm{CN}=4 ; 67,8.7 \%)$, and pentagonal $(\mathrm{CN}=5$; trigonal bipyramidal and square pyramidal; 60, 7.7\%) geometry. ${ }^{18}$

We have reduced each MOF crystal structure to its underlying topology and determined its net. A summary of our findings are presented in Figure 2 in which the CSD refcodes of the MOF crystals are matched to the building block shapes and the underlying net topology (note that for easy reference we have started a convention of giving each net its own arbitrary three letter designation, see Supporting Information and RCSR website http:/ / okeeffews1.la.asu.edu/RCSR/home.htm). It is remarkable that for each one of the building block geometries only one or two nets predominate in MOF chemistry. This is followed by a dramatic decease in the frequency of occurrence for other nets, albeit a significant number of these still occur in MOF structures.

Specifically, among 113 MOF structures with triangular (three-coordinated) building blocks, 72 (64\%) have the topology of srs $\left(\mathrm{Si}\right.$ in $\mathrm{SrSi}_{2}$ ) and $24(21 \%)$ have the ths topology ( $\mathrm{Si}$ in $\mathrm{ThSi}_{2}$ ). In the case of tetrahedral MOFs, there are a total of 335 structures. Of these, $233(70 \%)$ have the topology of dia (diamond) net, and only 31 (9\%) have that of sra $\left(\mathrm{Al}\right.$ in $\mathrm{SrAl}_{2}$ ). For MOF structures with square building blocks, there are a total of 47 structures, 21 (45\%) having the topology of the nbo (NbO) net, 18 (38\%) having the topology of the cds $\left(\mathrm{CdSO}_{4}\right)$ net, and $8(17 \%)$ having that of the lvt net. There are $67 \mathrm{MOF}$ structures that have mixed tetrahedral and square building blocks, 25 (37\%) having the topology of pts net (PtS), 13 (19\%) having that of mog (moganite) net, and 28 (42\%) having that of ptt net. For five-coordination, from a total of $60 \mathrm{MOF}$ structures, $28(47 \%)$ and $18(30 \%)$ have the topology of the bnn (BN) net and the sqp net, respectively. Finally, for octahedral building blocks, out of a total 152 structures, 145 (95\%) adopt the topology of the pcu (primitive cubic) net. We note that in the largest categories, those of triangular, tetrahedral, square, and octahedral geometries, the dominant nets are four out of the five regular nets, ${ }^{19}$ namely, srs, dia, nbo, and pcu; the fifth, bcu, is for eightcoordination (body-centered cubic).

It is clear from the preceding observations that among the infinite number of possible nets that could result from assembly of these shapes, only a handful are observed in the MOF crystal structures. Inspection of the symmetry of this subset of nets further revealed that they are all those with the highest possible symmetry at the vertex for the corresponding building block shape. ${ }^{20}$ We have identified two related quantities that appear to be particularly useful in developing hierarchical characterization of nets, namely, transitivity and order of point symmetry at the vertex. Associated with each net is a transitivity $p q$, which refers to $p$ kinds of vertex (joint) and $q$ kinds of edge (link, Figure 2). In general, small numbers for $p q$ are associated with higher symmetry. Thus if we determine the transitivity of the recurring nets in MOF structures, we find that the most symmetric (low transitivity) dominate (Figure 2). In the next section, we further relate the order of point symmetry at the vertex with frequency of net occurrence.

\section{Discovery of Inversion between Expected and Found Topologies}

Statistically one expects that in any system involving linking of shapes, the topological possibilities of least symmetry should vastly outnumber those of the highest symmetry, and thus the less symmetric topologies should predominate. ${ }^{21}$ However, our analysis of 3-D MOF structures in the CSD reveals an opposite trend. A summary of results for the case of tetrahedral nets (tetrahedral building blocks) is provided by a 3-D plot of order of point symmetry, $Z$ (number of vertices in the primitive cell), and frequency of occurrence for the theoretical and observed nets (Figure 3). On the top are the theoretically possible tetrahedral nets ${ }^{22}$ with one kind of vertex, and on the bottom is the analogous plot for what we have found for nets adopted by tetrahedral MOF structures. Note that most of the theoretical nets have lower order and relatively high $Z$ (low symmetry), but for MOF structures, one finds that those nets with high order and low $Z$ (high symmetry) are in fact the most frequently observed. How then do we explain this inversion? And what are the implications for the design of MOFs and other extended structures? We answer these questions below.

\section{Grammar for the Design and Construction of Extended Structures}

It is clear from the foregoing discussion that the manner in which MOFs have been synthesized is leading to the dominance of structures with nets of high symmetry. We believe that one of the reasons is that the components employed in the synthesis are simple. By this we mean that the typical preparation of a MOF involves reacting solutions containing a metal ion salt and an organic link, both components having maximum degrees of freedom and highly symmetric binding environment. The metal ion is spherical and nondirectional, and the link has welldefined points of contact that invariably are oriented at maximum vertex symmetry. Hence at the onset of the synthesis, the system is chemically and physically isotropic (this means unbiased and nondirectional), and indeed, under such conditions symmetrical organization is the most chemically reasonable way to satisfy metal coordination. This in turn leads one to expect that such highly symmetric building blocks would assemble to give MOF structures based on nets of the highest possible symmetry. In other words, when building blocks have sufficient flexibility and can in principle form many different extended structures because of coordination diversity of the metal ions, it is the highly symmetric nets that are most likely to form. We refer to these nets as "default" nets since they serve as the default selection in the assembly of nonrigid building blocks. These are the nets that Nature selects without built-in instructions to do otherwise (i.e., in the absence of design). On the basis of this argument, we propose that formation of structures 

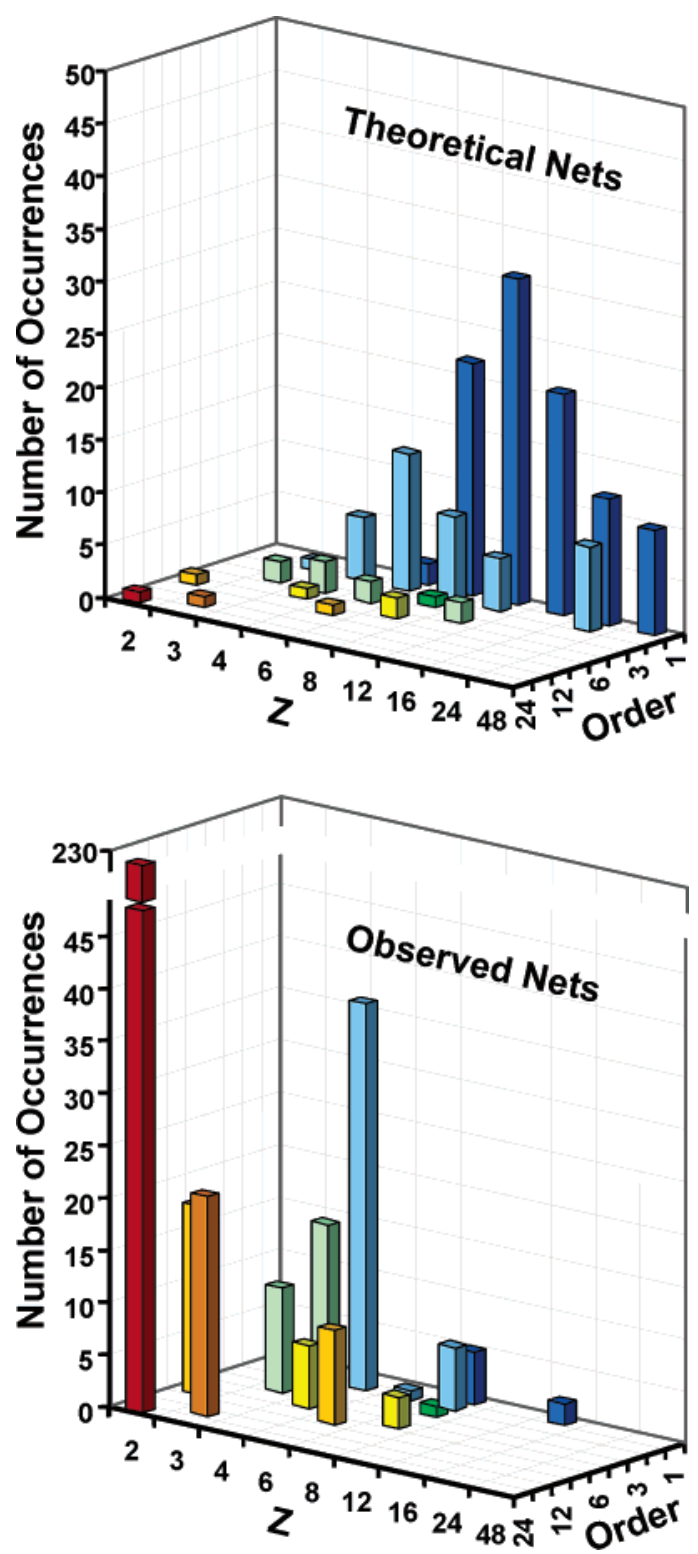

FIGURE 3. $3-D$ plots of $Z$ (number of vertices in the primitive cell), order (point group symmetry at the vertex), and frequency of occurrence shown here for tetrahedral nets to illustrate the presence of an inversion between theoretically expected nets that MOFs should adopt (top) and those actually observed (bottom).

based on nondefault nets requires the introduction of complexity (directional information) into the components of the synthesis.

Accordingly, we define several levels of complexity as illustrated in Figure 4. At the lowest level one (level 1) has free ions and links with highest possible degrees of freedom that lead to the default nets. At the next level, components consisting of objects such as (a) strong terminal ligands that compete with the link for coordination of metal ion, (b) an organic link with specialized shape (e.g., bent or twisted) as has been demonstrated for linked paddle wheels,,$^{15,23}$ and (c) a counterion or template the presence of which within the structure is necessary for its formation. Strictly speaking, structures produced within these two domains are not considered to be constructed by design because several possibilities still exist for a given set of building blocks to produce more than one net, albeit these possibilities are significantly reduced (especially in the second level) from the almost infinite number in the net space. We believe that a structure can be truly designed when a specific building block leads to a specific predetermined structure, which is the only possibility for that building block. Such structures fall into the highest level, which in essence conceives the construction of a net from a building block that codes specifically and only for that net.

To illustrate the prospects and challenges that exist at this level, we use the hypothetical structure known variously as "polybenzene" 24 or "cubic graphite". ${ }^{25}$ This material is a crystalline form of three-coordinated $\left(\mathrm{sp}^{2}\right)$ carbon in which all atoms are on six- and eight-membered rings that tile the $D$ periodic minimal surface. Electronic structure calculations show that it is best thought of as aromatic $\mathrm{C}_{6}$ rings linked by single bonds (hence the name).$^{24}$ Attractive features of the structure are its low energy (more stable than, for example, $\mathrm{C}_{60}$, ref 23) and its low-density open structure, which makes it an attractive candidate for applications such as gas storage. However, it seems clear that attempts to synthesize the structure by starting from units such as functionalized benzene rings would be doomed to failure, and indeed a successful synthesis is considered a formidable task. ${ }^{25}$ However, we have found that the structure can be deconstructed into units that have one eight- and four six-membered rings (left, Figure 4). In joining these, we note that (a) fourmembered rings are unlikely to form, (b) the presence of six- and eight-membered rings precludes formation of a planar structure such as graphite, and (c) joining such units (center and right, Figure 4) does in fact produce sixand eight-membered rings in the correct $2: 3$ ratio. We believe this is an achievable goal given the arsenal of $\mathrm{C}-\mathrm{C}$ bond forming reactions in organic chemistry that can be employed to accomplish the reticulation of those primary units. ${ }^{26}$

Although the polybenzene net is ideally suited for carbon, in principle, one can attempt to make a MOF structure based on this net by designing the primary units from metal-organic compositions. Obviously, in embarking on the synthesis of such building blocks, one would have to reckon with the fact that unlike carbon, control of the metal coordination geometry and stereochemistry will be far more difficult to achieve in the precise manner required to produce this net, and also the designer would need a good reason (property) to make such a net as a MOF.

This Account has shown that a large number of seemingly different MOF crystal structures are based on a handful of nets, which we refer to as default nets. These are the nets that are obtained when the building blocks are not specifically designed to achieve a particular preconceived net. The process by which we have reduced the crystal structures and assigned to them a net type leads to a taxonomy that helps the designer in interpreting and classifying the resulting structures. We have outlined a strategy based on three levels of complexity in which a 


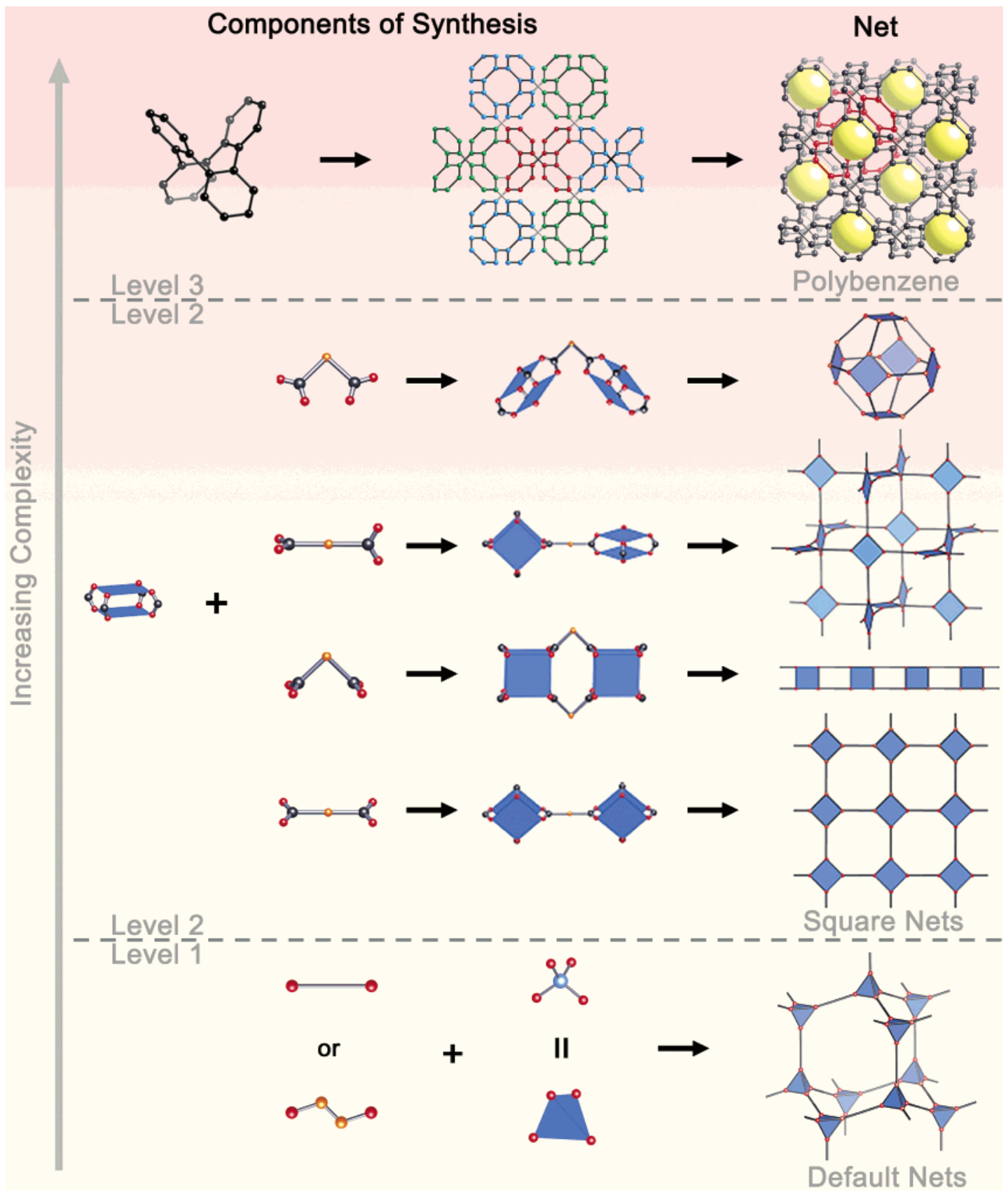

FIGURE 4. At level 1, a four-coordinated atom linked by ditopic linkers will almost invariantly form a structure based on diamond net. Level 2 gives examples of structures by design. Joining copper acetate paddlewheels by ditopic carboxylate linkers of the geometries shown schematically (the orange ball could stand for a phenyl group, for example) have the default structures shown (paddlewheels now shown as squares). All four (and others) have been achieved.,14 Level 3 shows the process of deconstruction of the polybenzene framework into "smart" building units (colored differently in the middle diagram) and the building up of a specific structure coded for by those building units. The yellow spheres represent the space that could be filled by guests.

system of grammar for the true design of structures can be used to achieve a specific structure by employing a building block that, at the highest level of complexity, codes specifically for that structure.

We conclude by remarking that in a famous essay ${ }^{27}$ Eugene Wigner expressed concern at "the unreasonable effectiveness of mathematics in the natural sciences". We who work in geometry do not share this concern; we feel that we discovered, rather than invented, the five regular nets. The present discovery that Nature uses these structures overwhelmingly in crystal topologies does not surprise us; rather it is the exceptions that are the occasions for thought.

We thank the NSF (M.O.'K and O.M.Y.; Grant DMR-0243082) and DOE (O.M.Y.) for partial funding of this research and Dr. Hashim M. Al-Hashimi (Department of Chemistry, University of Michigan) for his critical and insightful review of the manuscript.

Supporting Information Available: Methods of analysis, illustrations of the topologies with the arbitrary three-letter designations indicated, and tables including the vertex coordination numbers, total vertices, total edges, $Z$, order, net designations, transitivity 
number $(p q)$, space groups, unit cell parameters, and literature citations for the analyzed MOFs. This material is available free of charge via the Internet at http://pubs.acs.org.

\section{References}

(1) Winchester, S. The Map That Changed the World: William Smith and the Birth of Modern Geology; HarperCollins: New York, 2001.

(2) Using the QUEST software suite from the Cambridge Crystallographic Data Collection, we identified polymeric metal-organic structures using the simple search strings in concert with the polymeric screening methods *BTEST 53, 54. See also: Allen, F. The Cambridge Structure Database: a quarter of a million crystal structures and rising. Acta Crystallogr. 2002, B58, 380-388. Allen F., Motherwell, W. D. S. Applications of the Cambridge Structural Database in organic and crystal chemistry. Acta Crystallogr. 2002, B58, 407-422. Zorkii, P. M., Oleinikov, P. N. Crystal-chemical classes of "Cambridge" crystal structures: statistical analysis of topology. J. Struct. Chem. 2001, 42, 24-31.

(3) Yaghi, O. M.; O’Keeffe, M.; Ockwig, N. W.; Chae, H. K.; Eddaoudi, M.; Kim, J. Reticular design and the synthesis of new materials. Nature 2003, 423, 705-714.

(4) Cheetham, A. K.; Férey, G.; Loiseau, T. Open-framework inorganic materials. Angew. Chem., Int. Ed. 1999, 38, 3269-3292.

(5) Kitagawa, S.; Kitaura, R.; Noro, S. Functional porous materials. Angew. Chem., Int. Ed. 2004, 43, 2334-2375.

(6) Moulton, B.; Zaworotko, M. J. From molecules to crystal engineering: supramolecular isomerism and polymorphism in network solids. Chem. Rev. 2001, 101, 1629-1658.

(7) Holman, K. T.; Pivovar, A. M.; Ward, M. D. Engineering crystal symmetry and polar order in molecular host frameworks. Science 2001, 294, 1907-1911.

(8) Batten, S. R.; Robson, R. Interpenetrating nets: ordered, periodic entanglement. Angew. Chem., Int. Ed. 1998, 37, 1461-1494.

(9) Carlucci, L.; Ciani, G.; Proserpio, D. M. Polycatination, polythreading and polyknotting in coordination network chemistry. Coord. Chem. Rev. 2003, 246, 247-289.

(10) Researchers often will mention the "design" of a structure, but in fact, we know little about what structures might result from the assembly of specific building units. The automated assembly of secondary building units (AASBU) method uses computer simulation to predict a priori which structures will form from specific building units. See especially: Mellot-Draznieks, C. Dutour, J.; Férey, G. Hybrid Organic-Inorganic Frameworks: Routes for Computational Design and Structure Prediction. Angew. Chem., Int. Ed. 2004, 43, 6290-6296.

(11) Corey, E. J.; Cheng, X.-M. The Logic of Chemical Synthesis; WileyInterscience: New York, 1995.

(12) Chen, B.: Ockwig, N. W.; Fronczek, F. R.; Contreras, D. S.; Yaghi, O. M. Transformation of a Metal-Organic Framework from the $\mathrm{NbO}$ to PtS Net Inorg. Chem., in press.

(13) Eddaoudi, M.; Moler, D. B.; Li, H. L.; Chen, B. L.; Reineke, T. M. O'Keeffe, M.; Yaghi, O. M. Modular Chemistry: Secondary Building Units as a Basis for the Design of Highly Porous and Robust
Metal-Organic Carboxylate Frameworks. Acc. Chem. Res. 2001, 34, 319-330.

(14) Kim, J.; Chen, B. L.; Reineke, T. M.; Li, H. L.; Eddaoudi, M.; Moler, D. B.; O'Keeffe, M.; Yaghi, O. M. Assembly of metal-organic frameworks: structure design and sorption properties. J. Am. Chem. Soc. 2001, 123, 8239-8247.

(15) Eddaoudi, M.; Kim, J.; Vodak, D.; Sudik, A.; Wachter, J.; O’Keeffe M.; Yaghi, O. M. Supramolecular chemistry and self-assembly special feature: geometric requirements and examples of important structures in the assembly of square building blocks. Proc. Natl. Acad. Sci. U.S.A. 2002, 99, 4900-4904.

(16) Structure reduction performed using the Cesius ${ }^{2}$ Software suite. Complete structure reduction details are provided in the Supporting Information.

(17) Delgado-Friedrichs, O.; O'Keeffe, M. Identification of and symmetry computation for crystal nets. Acta Crystallogr. 2003, A59, $351-360$.

(18) For articles that report a series of compounds that have the same underlying topology (isoreticular), we counted such compounds as a single occurrence; however, the trend remains unchanged even when the total number of compounds belonging to such isoreticular series is counted as multiple occurrences.

(19) Delgado-Friedrichs, O.; O'Keeffe, M.; Yaghi, O. M. Three-periodic nets and tilings: regular and quasiregular nets. Acta Crystallogr. 2003, A59, 22-27. Of course these nets were known long before our work. Our contribution is the recognition of the concept of regularity as applied to nets and the demonstration that there are just five nets with this property.

(20) We note that a net of high symmetry like that of diamond may be the underlying topology of a MOF structure that actually has low crystallographic symmetry. It is the high intrinsic (combinatorial) symmetry of the net that is relevant to our classification system.

(21) Thus it is known that "almost" all polyhedra are asymmetric: Bender, E. A.; Wormald, N. C. Almost all convex polyhedra are asymmetric. Can. J. Math. 1985, 37, 854-871.

(22) O'Keeffe, M.; Brese, N. E. Uninodal 4-connected 3D nets. I. Nets without 3- or 4- rings. Acta Crystallogr. 1992, A48, 663-669 and unpublished.

(23) Cotton, F. A.; Lin, C.; Murillo, C. A. Supramolecular arrays based on dimetal building units. Acc. Chem. Res. 2001, 34, 759-771.

(24) O'Keeffe, M.; Adams, G. B.; Sankey, O. F. Predicted new low energy forms of carbon. Phys. Rev. Lett. 1992, 68, 2325-2328.

(25) Shen, X.; Ho, D. M.; Pascal, R. A., Jr. Synthesis of polyphenylene dendrimers related to "cubic graphite". J. Am. Chem. Soc. 2004, $126,5798-5805$

(26) An elegant illustration of how this was done for the analogous molecular chemistry has appeared in a recent report on the stepby-step synthesis of $\mathrm{C}_{60}$ : Scott, L. T.; Boorum, M. M.; McMahon B. J.; Hagen, S.; Mack, J.; Blank, J.; Wegner, H.; de Meijere, A. A rational chemical synthesis of $C_{60}$. Science 2002, 295, 1500-1503.

(27) Wigner, E. P. The unreasonable effects of mathematics in the natural sciences. Commun. Pure Appl. Math. 1960, 13, 1-14. 